\title{
Noninvasive Assessment of Foot Perfusion in a Rabbit Model of Atherosclerosis Using Dynamic Volume Perfusion CT with an Upslope Method
}

Hooney Min

Seoul National University Bundang Hospital

Saebeom Hur

Seoul National University Hospital

Jae Hwan Lee ( $\sim$ lzhwanmd@gmail.com )

Seoul National University Bundang Hospital

Chang Jin Yoon

Seoul National University Bundang Hospital

Won Seok Choi

Seoul National University Bundang Hospital

\section{Seunghyun Lee}

Seoul National University Hospital

\section{Research Article}

Keywords: Angiography, Animals, Contrast Media, Feasibility Studies, Foot / blood supply*, Foot / diagnostic imaging*, Peripheral Vascular Diseases / diagnostic imaging*, Rabbits, Radiographic Image Interpretation, Computer-Assisted, Regional Blood Flow, Tomography, X-Ray Computed / methods

Posted Date: September 1st, 2021

DOI: https://doi.org/10.21203/rs.3.rs-829118/v1

License: (a) (1) This work is licensed under a Creative Commons Attribution 4.0 International License. Read Full License 


\section{Abstract}

Objectives: To evaluate the feasibility of foot dynamic volume CT with the upslope method and to demonstrate macrovascular reactivity and microvascular perfusion during cuff-induced reactive hyperemia state in a rabbit model of atherosclerosis.

Materials and Methods: 30 New Zealand male rabbits were divided into 2 groups: dietary hypercholesterolemia induced atherosclerosis $(n=10)$ and normal diet control $(n=20)$. To measure for macrovascular reactivity, perfusion parameters of the left posterior tibial artery was measured at baseline and at reactive hyperemia state. For the evaluation of microvascular perfusion, color-coded perfusion map of the plantar dermis was generated for perfusion CT scan by an in-house dedicated analysis software based on upslope method. Dermal perfusion values were measured and analyzed before and after cuff-induced reactive hyperemia.

Results: Foot dynamic volume CT with the upslope method demonstrated significant impairment of both macrovascular reactivity and microvascular perfusion in atherosclerotic rabbits during cuff-induced reactive hyperemia $(\mathrm{CRH})$ state. Arterial time-to-peak of cholesterol-fed rabbits failed to show acceleration while healthy rabbits showed significant decrease in time. Microvascular perfusion calculated by perfusion value $(P<0.01)$ and perfusion ratio $(P=.014)$ showed decreased microvascular perfusion in cholesterol fed rabbits compared to healthy rabbits during $\mathrm{CRH}$ state. Post-CT pathologic examination revealed decreased endothelial cell density in cholesterol-fed rabbits $(P<0.001)$.

Conclusions: Foot perfusion CT using upslope method provides perfusion parameters for large arteries and a perfusion map of the foot during cuff-induced reactive hyperemia in rabbit models of atherosclerosis. It may be a useful tool for the quantitative and functional evaluation of atherosclerotic peripheral arterial disease.

\section{Summary Statement}

Dynamic volume CT with upslope method provides a perfusion map of the foot and reveals impairment during reactive hyperemia in a rabbit model of atherosclerosis.

\section{Key Results}

- Dynamic volume CT with upslope method was used to generate a perfusion map to evaluate macrovascular reactivity and microvascular perfusion in a rabbit model of atherosclerosis.

- During cuff-induced reactive hyperemia state, rabbit models of atherosclerosis showed a higher arterial time-to-peak in its tibial artery compared to healthy rabbits, suggesting deterred vasoreactivity of large arteries.

- Compared to healthy rabbits, atherosclerosis group showed impaired perfusion in the foot during cuff-induced reactive hyperemia state. 


\section{Introduction}

Atherosclerotic peripheral arterial disease (PAD) is a global health problem, afflicting over 200 million people worldwide ${ }^{1-3}$. PAD is the third leading cause of atherosclerotic cardiovascular morbidity, following coronary artery disease and stroke ${ }^{1}$. Disability and mortality associated with PAD has significantly increased over the last 20 years ${ }^{4}$.

Ankle brachial index $(A B I)$ is the most readily available, and validated method to screen and diagnose $P A D^{5}$. ABI relies on macroscopic measurements of pressure gradient in the limbs to provide important diagnostic and prognostic information, such as risk of death, myocardial infarction and stroke ${ }^{67}$. Yet, despite the diagnostic and prognostic utility, $A B I$ does not give any anatomical information. It is also not applicable to evaluate non-compressible arteries with heavy calcification or below-ankle diseases 8,9 . Other modalities such as peripheral artery tonometry (PAT) or flow mediated dilatation (FMD), while limited in use, can evaluate macrovascular reactivity but do not provide any anatomical information. Modalities that provide anatomic information for the evaluation of PAD such as nuclear imaging, computed tomographic (CT) angiography, magnetic resonance (MR) angiography, and conventional

angiography visualize macrovascular lesions but do not evaluate microvascular perfusion ${ }^{10-12}$. The ideal test for PAD would provide both macrovascular and microvascular information as well as anatomical details to assess disease severity, guide decision-making, and predict treatment response. Therefore, a pragmatic modality to concurrently evaluate arterial blood flow, microvascular foot perfusion and anatomic lesions is imperative.

Perfusion imaging has been widely used to diagnose and treat coronary artery diseases and cerebral ischemic diseases ${ }^{13-15}$. In evaluating cerebral ischemic diseases, perfusion CT or MRI allows for the visualization of ischemic penumbra zones, providing practical information on brain tissue viability 13,14 . In the evaluation of coronary artery disease, the concept of "myocardial perfusion reserve" allows for the evaluation of the surrounding microvasculature's ability to meet tissue demands upon stressors ${ }^{16}$. Perfusion reserve, defined as the ratio of global blood flow at stress vs. rest, allows for identification of atrisk tissue. Widely used in the clinic, stress testing with adenosine can induce vasodilatation and hyperemia which allows for the identification of myocardial tissue with hypoperfusion in asymptomatic individuals without abnormalities on conventional angiography ${ }^{16}$. These imaging modalities provide important information to non-invasively identify areas at risk and guide treatment. However, perfusion imaging modalities have yet to be applied for the evaluation of the foot for patients with atherosclerosis or claudication but no significant artery occlusion.

In our previous study, we showed that foot perfusion CT with the upslope method can provide both qualitative and quantitative assessment of the foot in patients with PAD ${ }^{17}$. In addition, we identified the presence of a dermal hyperperfusion band in the rabbit's feet that served as the region of interest in evaluating foot tissue perfusion ${ }^{17}$. 
Here, we evaluated the feasibility of dynamic volume CT with the upslope method during cuff-induced hyperemia, to demonstrate macrovascular reactivity and microvascular perfusion in a rabbit model of atherosclerosis.

\section{Materials And Methods}

\section{Statement}

All experiments and methods were performed in accordance with relevant guidelines and regulations. All experimental protocols were approved by a named institutional/licensing committee. Specifically, animal research protocol was approved by Institutional Animal Care and Use Committee of Seoul National University Bundang Hospital. The study was carried out in compliance with the ARRIVE guidelines. All animals were euthanized with an intravenous injection of a lethal amount $(7-10 \mathrm{ml})$ of xylazine hydrochloride under deep anesthesia.

\section{Animal Model Preparation}

Thirty New Zealand white male rabbits weighting 3000-3500 g were used in our study. All animals were housed in cages with a 12-hour, light/dark cycle and ad libitum access to water. For anesthesia, $5 \mathrm{mg} / \mathrm{kg}$ body weight tiletamine-zolazepam (Zoletil 50; Virbac, Carros, France) and $2 \mathrm{mg} / \mathrm{kg}$ body weight of $2 \%$ xylazine hydrochloride (Rompun; Bayer, Seoul, Republic of Korea) were injected intramuscularly in the posterior thigh. Atherosclerosis was induced as described in previous studies ${ }^{18-22} .10$ rabbits in the atherosclerosis group were fed an atherogenic diet consisting of $0.5 \%$ cholesterol and $6 \%$ peanut oil for 5 weeks and then switched to a diet containing lower cholesterol $(0.025 \%)$ for another 5 weeks to prevent liver failure ${ }^{18,22}$. The 20 rabbits in the control group (group B) were fed normal rabbit chow for all study periods. Study protocol for induction of atherosclerosis is schematically shown in Figure 1.

\section{Angiography}

All rabbits underwent angiography to evaluate the presence of significant stenosis or occlusion of bilateral aortoiliac and femorotibial arteries, one day prior to CT scan. After sedation, rabbits were placed in supine position and right central ear artery of each rabbit was cannulated using 16 gauze IV cannula. To access the infrarenal aorta, a 2.0-Fr microcatheter (progreat a, Terumo, Tokyo, Japan) was advanced via the ear artery to the iliofemoral artery and femorotibial angiography was performed.

\section{Foot Perfusion CT}

Perfusion CT protocol similar to previous experiment was performed to evaluate foot perfusion status of each rabbit as detailed in a previous study ${ }^{1717}$. In brief, each animal was laid down on the table of a 64 - 
detector CT scanner (Ingenuity; Philips Medical, Einthoven, the Netherlands) with 4-cm z-axis coverage in volume scan mode. First, baseline perfusion CT scan was obtained by using following parameters: scan coverage including the whole hindfoot and ankle, volume scan mode without table movement, gantry rotation time of $400 \mathrm{msec}$, detector collimation of $64 \times 0.625 \mathrm{~mm}$, tube voltage of $100 \mathrm{kV}$, and tube current of $100 \mathrm{~mA}$. The dynamic volume scanning started 1-3 seconds after intravenous bolus injection of $3 \mathrm{~mL}$ of iopamidol (Pamiray, 370mg of iodine per milliliter; Dongkook Pharmaceutical, Seoul, South Korea) contrast material. Contrast material injection was followed by injection of an equal volume of normal saline, both injected with an automated dual-rail injector (Stellant; Medrad, Warrendale, $\mathrm{Pa}$ ) at a rate of $1 \mathrm{~mL} / \mathrm{sec}$ via the left auricular vein. After 5 minutes of rest, a disposable neonatal blood pressure cuff (Welch Allyn, NY, USA) was applied to animal's left thigh and cuff was inflated to $200 \mathrm{mmHg}$ to block blood flow to left foot for 3 minutes. As the cuff deflated to $0 \mathrm{mmHg}$ within 3 seconds, a second

perfusion CT was obtained in the same manner as the previous scan ${ }^{23-26}$. Perfusion CT protocol timeline was illustrated in Figure 1.

\section{Image reconstruction and analysis of perfusion CT findings}

All the CT images were transferred to an image archiving and communications workstation (INFINITT; Infinitt healthcare, Seoul, Republic of Korea) and analyzed by in-house perfusion analysis software ${ }^{17}$. The image reconstruction method for the perfusion CT data was identical to that of a prior animal experiment ${ }^{17}$, except that the plantar dermis in the heel base rather than that of the toes was used as the representative tissue to set the upslope time range. Each session of perfusion CT data was sent to the software and a pixel-based, color-coded perfusion map was generated by using the upslope method ${ }^{17}$. Based on a perfusion map, region of interest (ROI) for posterior tibial artery and plantar dermis was placed, and time-attenuation curve ${ }^{1}$ was calculated respectively (Fig 1). Upslope time range was set from the arterial time-attenuation curve and the following perfusion parameters were obtained; Peak arterial enhancement (PAE), Time to peak enhancement (TTP), and maximal upslope (m). Time attenuation curve of each pixel was normalized by the maximal arterial enhancement value. Quantitative analysis of perfusion parameters was performed by two radiologists (S.H and H.D.M) in consensus. Regional blood flow at baseline perfusion CT scan was measured at the dermis of the foot sole by placing an ROI on the axial image. In the same way, ROI was set in the corresponding location of the plantar dermis on the perfusion map obtained from the second CT scan, and each perfusion value and the ratio before and after inducing $\mathrm{CRH}$ was calculated.

\section{Pathological analysis}

All animals were preanesthesized and sacrificed with an intravenous injection of xylazine hydrochloride. Dermal tissues of left heel of all rabbits were harvested. Tissues obtained were weighed and half of the tissues were processed to evaluate number of each color's microsphere, the other specimens were fixed in $10 \%$ buffered formalin and were embedded in paraffin. Thereafter, tissue samples were treated with 
hematoxylin and eosin staining for basic histopathological examinations and consecutive section was treated CD 31 staining (DAKO Corp., Carpinateria, CA, USA) for microvascular density (MVD) evaluation. After digital images of the histologic slides were obtained (Leica Microsystems, Mannheim, Germany), MVD was calculated using image analysis software (Image J, version 1.45s; National Institutes of Health, Bethesda, MD) (11). The five hot spots with the most intense vascularization were selected by L.J.H who was blinded to information of animal group, while screening at a low-power field $(\times 40)$. MVD counts of the five areas per the hot spots were performed at a high-power field (S.B.C) $(\times 100)$. Any brown-stained endothelial cells or endothelial cell clusters clearly separated from adjacent microvessels were counted as one microvessel stained by the anti-CD31 antibody, irrespective of the presence of a vessel lumen. The mean microvessel area percentage of all the measured areas was determined to be the MVD.

\section{Statistical analysis}

All data in the study was reported as mean \pm SD. The perfusion value and ratio of regional blood flow between baseline and $\mathrm{CRH}$ in each group was tested by using paired-T test and Wilcoxon signed rank test. The value of change of each parameter between two groups were compared using repeated measures analysis of variance test. The data processing and analysis were performed using Statistical Package for the Social Sciences version 18.0 (SPSS Inc, IBM Company, Chicago, IL). A two-sided P value of less than 0.05 indicates that the groups differ significantly in terms of statistical results.

\section{Results}

\section{Angiography and Foot perfusion CT}

Angiography was performed successfully in all rabbits, and there were no significant stenosis or occlusions in the aortoiliac and femorotibial arteries in both groups (Fig 2). Foot perfusion CT was successfully performed in all rabbits from both groups at baseline and after cuff-induced reactive hyperemia $(\mathrm{CRH})$ state.

\section{Quantitative analysis of arterial perfusion parameters at baseline and cuff-induced reactive hyperemia}

Serial measurements of arterial perfusion parameters were taken at baseline and $\mathrm{CRH}$ phase in both the healthy and the cholesterol-fed groups. At baseline, cholesterol-fed rabbits compared to healthy rabbits showed increased PAE, increased maximum arterial upslope, $m$ but no differences in TTP (cholesterol-fed vs healthy rabbits; $354.6 \pm 96.47 \mathrm{HU}$ vs $255.51 \pm 96.22 \mathrm{HU}, P<0.001 ; 109.30 \pm 25$ vs $57.35 \pm 33.44 \mathrm{HU}, P<$ $0.001 ; 10.7 \pm 1.82 \mathrm{HU}$ vs $11.65 \pm 2.32 \mathrm{HU}, P=0.269$, respectively) (Fig 3 ). During $\mathrm{CRH}$ phase, both groups showed an increase in PAE and $m$ compared to baseline, but intergroup differences were not significant $(P=.922$ and $P=.977$, respectively) (Fig 3$)$. However, the arterial TTP of the healthy group showed 


\section{Dermal perfusion value and perfusion ratio analysis between baseline and cuff-induced reactive hyperemia}

Figure 4 summarizes the result of perfusion values of left plantar dermis in the two groups at baseline and $\mathrm{CRH}$ phase. Perfusion values were calculated as PAE multiplied by heal-base perfusion. There was no significant difference of dermal perfusion value between healthy group and cholesterol-fed group at baseline (35040.61 \pm 17750.58 vs. $47427.59 \pm 18968.08$; $P=0.57$ ) (Fig 4a). Dermal perfusion value of healthy rabbits significantly increased on $\mathrm{CRH}$ phase $(35040.61 \pm 17750.58$ to $57582.43 \pm 22585.11 \mathrm{HU}$; $P=0.008$ ), whereas no such surge was demonstrated in cholesterol-fed groups (47427.59 \pm 18968.08 to $46692.38 \pm 19797.93 \mathrm{HU} ; P=0.878$ ) (Fig 4a). The perfusion ratio (dividing perfusion values of $\mathrm{CRH}$ to that of baseline) of left foot for normal rabbits were significantly higher than that of cholesterol-fed rabbits (1.62 \pm 0.74 vs. $0.99 \pm 0.18 ; P=0.014)$ (Fig 4b).

\section{Endothelial cell density between healthy and cholesterol-fed groups}

All animals were sacrificed post CT scans and foot tissue was pathologically examined for differences in endothelial cell density. Endothelial cell density as measured by CD 31 showed decreased endothelial density in cholesterol fed rabbits ( $2.22 \pm 0.24$ vs. $0.69 \pm 0.04 ; P<0.001)$ (Fig 5.).

\section{Discussion}

The present study showed that the combination of dynamic volume perfusion foot CT with the upslope method and cuff-induced reactive hyperemia (CRH) was a feasible method for evaluating macrovascular reactivity and microvascular perfusion in a rabbit model of atherosclerosis. Using this combination, impairment of macrovascular reactivity and microvascular perfusion of the cholesterol-fed rabbits were non-invasively and quantitatively demonstrated.

In this study, cholesterol-fed rabbits compared to healthy rabbits showed increased PAE, increased maximum arterial upslope, $m$ but no differences in TTP. However, during CRH phase, the atherosclerosis rabbits showed a hindered vasoreactivity as implied by the delayed arterial TTP. A delayed TTP on reactive hyperemia state implies that the target artery has lost appropriate reactivity to hyperemia leading to hypoperfusion. As atherosclerotic burden progresses, arterial TTP decreases reflecting disease severity ${ }^{27-30}$. Our previous study showed that decreased arterial TTP of patients with PAD was improved after revascularization therapy ${ }^{17}$. 
Perfusion value (peak arterial enhancement multiplied by heal based perfusion), a surrogate measure of autoregulation, increased during $\mathrm{CRH}$ phase compared to baseline for healthy rabbits. However, this autoregulation did not occur in the atherosclerotic rabbits. We hypothesized that the lack of autoregulation without any significant stenosis or occlusions in the atherosclerotic rabbits was due to endothelium dysfunction. Previous studies show that endothelium dysfunction occurs even before any apparent structural changes to vessel walls are detected on angiography or ultrasound ${ }^{31}$. Studies with acetylcholine test or measurements of FMD detected endothelial dysfunction at both conduit and microvascular levels in patients with coronary risk factors but no structural evidence on angiography or ultrasound ${ }^{32,33}$. Our study confirmed that cholesterol-fed rabbits had a lower endothelial cell density, suggesting possible endothelium dysfunction as mechanism for impaired autoregulation during $\mathrm{CRH}$ phase.

Foot perfusion ratio decreased in the atherosclerotic rabbit compared to those of healthy rabbits. As perfusion ratio reflects surrounding microvasculature's ability to meet tissue demands upon hyperemia, combined dynamic foot perfusion CT with $\mathrm{CRH}$ can be used to assess 'vulnerable areas' to ischemic injury. In cardiac MRI adenosine stress test, perfusion ratio, more often referred to as 'myocardial perfusion reserve', is already utilized to evaluate the extent of ischemic injury in myocardial infarctions (16).

There are few studies evaluating foot perfusion using CT imaging, but these studies target patients with overt macrovascular dysfunction such as critical limb ischemia ${ }^{10,34}$. The present study shows the feasibility of dynamic volume perfusion foot CT to detect early microvascular dysfunction in atherosclerosis model without macrovascular stenosis or occlusion, non-invasively and quantitatively. This is particularly important considering that currently available tools fail to provide information regarding anatomical area of decreased microperfusion, and monitor changes of microcirculation after treatment.

This study has several limitations. The rabbit model of atherosclerosis may have inherent differences in pathophysiology to human atherosclerosis as atherosclerosis is a multifactorial disease developed over a long time period with species-to-species variation. In addition, our rabbit model of atherosclerosis did not have significant macrovascular lesions in the large arteries which is found in human patients with peripheral artery diseases. However, the lack of macrovascular lesion allowed us to measure and compare TTP and perfusion parameters in the dermis, identifying possible early changes of atherosclerosis. Lastly, this study was also limited in only measuring one areas of foot perfusion due to the small size of the rabbit feet and a single dominant artery in the rabbit.

In conclusion, macrovascular reactivity and microvascular perfusion can be measured in a rabbit model of atherosclerosis by combination of perfusion foot CT using the upslope method and CRH. TTP in the tibial artery was prolonged during cuff-induced reactive hyperemic state in the atherosclerotic rabbits compared to healthy rabbits suggesting delayed vasoreactivity. Microvascular perfusion parameters such as perfusion value and perfusion ratio showed impairment in the atherosclerotic rabbits, suggesting ways 
to detect at-risk tissues and early manifestation of atherosclerosis. Further human studies are warranted to clinically validate the usefulness of foot perfusion CT with $\mathrm{CRH}$.

\section{Abbreviations}

$\mathrm{CRH}=$ Cuff-induced Reactive Hyperemia, PAE $=$ Peak Arterial Enhancement, TTP = Time to Peak

\section{Declarations}

\section{Author contributions}

SH and JHL conceived of and designed the project. JHL conducted all wet lab work. CJY and WSC provided conceptual advice on analysis of data. HDM and JHL analyzed and interpreted the data. HDM, $\mathrm{SH}, \mathrm{JHL}$ and SL prepared the manuscript, with contributions from all authors. All authors read and approved the final manuscript.

\section{Competing interests}

The authors declare no competing interests.

\section{Funding Information:}

This work was supported by Seoul National University Bundang hospital research fund [14-2020-005], and Korea Health Technology R\&D Project

[HI18C0493] funded by the Ministry of Health \& Welfare, and grant no. NRF2015R1C1A1A02036761 from the Basic Science Research Program of the National Research Foundation of Korea, which is funded by the Ministry of Science, ICT and Future Planning. The funders had no role in study design, data analysis, or preparation of the manuscript.

\section{References}

1. Alvelo, J. L. et al. Radiotracer Imaging Allows for Noninvasive Detection and Quantification of Abnormalities in Angiosome Foot Perfusion in Diabetic Patients With Critical Limb Ischemia and Nonhealing Wounds. Circ Cardiovasc Imaging, 11, e006932 https://doi.org/10.1161/CIRCIMAGING.117.006932 (2018).

2. Fowkes, F. G. et al. Comparison of global estimates of prevalence and risk factors for peripheral artery disease in 2000 and 2010: a systematic review and analysis. Lancet (London, England), 382, 1329-1340 https://doi.org/10.1016/S0140-6736(13)61249-0 (2013). 
3. Dormandy, J. A. \& Rutherford, R. B. Management of peripheral arterial disease (PAD). TASC Working Group. TransAtlantic Inter-Society Consensus (TASC). Journal of vascular surgery, 31, S1-S296 (2000).

4. Sampson, U. K. et al. Global and regional burden of death and disability from peripheral artery disease: 21 world regions, 1990 to 2010 . Glob Heart 9, 145-158 e121, doi:10.1016/j.gheart.2013.12.008 (2014).

5. Misra, S. et al. Perfusion Assessment in Critical Limb Ischemia: Principles for Understanding and the Development of Evidence and Evaluation of Devices: A Scientific Statement From the American Heart Association., 140, e657-e672 https://doi.org/10.1161/CIR.0000000000000708 (2019).

6. Ankle, B. et al. Ankle brachial index combined with Framingham Risk Score to predict cardiovascular events and mortality: a meta-analysis. JAMA, 300, 197-208 https://doi.org/10.1001/jama.300.2.197 (2008).

7. Faglia, E. et al. Predictive values of transcutaneous oxygen tension for above-the-ankle amputation in diabetic patients with critical limb ischemia. European journal of vascular and endovascular surgery: the official journal of the European Society for Vascular Surgery, 33, 731-736 https://doi.org/10.1016/j.ejvs.2006.12.027 (2007).

8. Shishehbor, M. H. \& Bunte, M. C. Time to Redefine Critical Limb Ischemia. JACC. Cardiovascular interventions, 10, 2317-2319 https://doi.org/10.1016/j.jcin.2017.09.012 (2017).

9. Bunte, M. C., Jacob, J., Nudelman, B. \& Shishehbor, M. H. Validation of the relationship between ankle-brachial and toe-brachial indices and infragenicular arterial patency in critical limb ischemia. Vasc Med, 20, 23-29 https://doi.org/10.1177/1358863X14565372 (2015).

10. Barfett, J., Velauthapillai, N., Kloeters, C., Mikulis, D. J. \& Jaskolka, J. D. An en bloc approach to CT perfusion for the evaluation of limb ischemia. The international journal of cardiovascular imaging, 28, 2073-2083 https://doi.org/10.1007/s10554-011-9978-z (2012).

11. Bajwa, A. et al. Assessment of tissue perfusion in the lower limb: current methods and techniques under development. Circ Cardiovasc Imaging, 7, 836-843 https://doi.org/10.1161/CIRCIMAGING.114.002123 (2014).

12. Benitez, E., Sumpio, B. J., Chin, J. \& Sumpio, B. E. Contemporary assessment of foot perfusion in patients with critical limb ischemia. Seminars in vascular surgery, 27, 3-15 https://doi.org/10.1053/j.semvascsurg.2014.12.001 (2014).

13. Latchaw, R. E. et al. Guidelines and recommendations for perfusion imaging in cerebral ischemia: A scientific statement for healthcare professionals by the writing group on perfusion imaging. from the Council on Cardiovascular Radiology of the American Heart Association. Stroke, 34, 1084-1104 https://doi.org/10.1161/01.STR.0000064840.99271.9E (2003).

14. Demeestere, J., Wouters, A., Christensen, S., Lemmens, R. \& Lansberg, M. G. Review of Perfusion Imaging in Acute Ischemic Stroke: From Time to Tissue., 51, 1017-1024 https://doi.org/10.1161/STROKEAHA.119.028337 (2020). 
15. Sandfort, V., Lima, J. A. \& Bluemke, D. A. Noninvasive Imaging of Atherosclerotic Plaque Progression: Status of Coronary Computed Tomography Angiography. Circ Cardiovasc Imaging, 8, e003316 https://doi.org/10.1161/CIRCIMAGING.115.003316 (2015).

16. Bonetti, P. O. et al. Noninvasive identification of patients with early coronary atherosclerosis by assessment of digital reactive hyperemia. Journal of the American College of Cardiology, 44, 2137$2141 \mathrm{https} / / /$ doi.org/10.1016/j.jacc.2004.08.062 (2004).

17. Hur, S. et al. Quantitative Assessment of Foot Blood Flow by Using Dynamic Volume Perfusion CT Technique: A Feasibility Study., 279, 195-206 https://doi.org/10.1148/radiol.2015150560 (2016).

18. Yanni, A. E. Laboratory rabbit and high-cholesterol diet: what is taken for granted may not be so simple. Laboratory animals, 48, 349-350 https://doi.org/10.1177/0023677214544184 (2014).

19. Mateo, J., Izquierdo-Garcia, D., Badimon, J. J., Fayad, Z. A. \& Fuster, V. Noninvasive assessment of hypoxia in rabbit advanced atherosclerosis using (1)(8)F-fluoromisonidazole positron emission tomographic imaging. Circ Cardiovasc Imaging, 7, 312-320

https://doi.org/10.1161/circimaging.113.001084 (2014).

20. Khanna, V. et al. Cholesterol Diet Withdrawal Leads to an Initial Plaque Instability and Subsequent Regression of Accelerated Iliac Artery Atherosclerosis in Rabbits. PloS one, 8, e77037 https://doi.org/10.1371/journal.pone.0077037 (2013).

21. Nakazawa, G. et al. Evaluation of Polymer-Based Comparator Drug-Eluting Stents Using a Rabbit Model of Iliac Artery Atherosclerosis. Circ-Cardiovasc Inte, 4, 38-75 https://doi.org/10.1161/Circinterventions.110.957654 (2011).

22. Fan, J. et al. Rabbit models for the study of human atherosclerosis: From pathophysiological mechanisms to translational medicine. Pharmacology \& therapeutics 146C, 104-119, doi:10.1016/j.pharmthera.2014.09.009 (2015).

23. Lekakis, J. et al. Methods for evaluating endothelial function: a position statement from the European Society of Cardiology Working Group on Peripheral Circulation. Eur J Cardiov Prev R, 18, 775-789 https://doi.org/10.1177/1741826711398179 (2011).

24. Higashi, Y. Assessment of endothelial function. History, methodological aspects, and clinical perspectives. International heart journal, 56, 125-134 https://doi.org/10.1536/ihj.14-385 (2015).

25. Matsuzawa, Y., Kwon, T. G., Lennon, R. J., Lerman, L. O. \& Lerman, A. Prognostic Value of FlowMediated Vasodilation in Brachial Artery and Fingertip Artery for Cardiovascular Events: A Systematic Review and Meta-Analysis. Journal of the American Heart Association, 4, https://doi.org/10.1161/jaha.115.002270 (2015).

26. Guo, Z. et al. Hepatic resection or transarterial chemoembolization for hepatocellular carcinoma within Milan criteria: A propensity score matching analysis., 96, e8933 https://doi.org/10.1097/MD.0000000000008933 (2017).

27. Breslau, P. J., Slot, H. B. \& Greep, J. M. Comparative study of strain gauge plethysmography and Doppler ultrasound in patients with occlusive arterial disease of the lower extremities., 32, 840-845 https://doi.org/10.1177/000331978103201206 (1981). 
28. Davies, W. T. Blood flow measurement in patients with intermittent claudication., 31, 164-175 https://doi.org/10.1177/000331978003100303 (1980).

29. Myers, K. The Investigation of Peripheral Arterial Disease by Strain Gauge Plethysmography., 15 , 293-304 https://doi.org/10.1177/000331976401500701 (1964).

30. Tonnesen, K. H. Muscle blood flow during exercise in intermittent claudication. Validation of the 133xenon clearance technique: clinical use by comparison to plethysmography and walking distance., 37, 402-410 https://doi.org/10.1161/01.cir.37.3.402 (1968).

31. Davignon, J. \& Ganz, P. Role of endothelial dysfunction in atherosclerosis., 109, III27-32 https://doi.org/10.1161/01.CIR.0000131515.03336.f8 (2004).

32. Reddy, K. G., Nair, R. N., Sheehan, H. M. \& Hodgson, J. M. Evidence that selective endothelial dysfunction may occur in the absence of angiographic or ultrasound atherosclerosis in patients with risk factors for atherosclerosis. J Am Coll Cardiol, 23, 833-843 https://doi.org/10.1016/07351097(94)90627-0 (1994).

33. Celermajer, D. S., Sorensen, K. E., Bull, C., Robinson, J. \& Deanfield, J. E. Endothelium-dependent dilation in the systemic arteries of asymptomatic subjects relates to coronary risk factors and their interaction. J Am Coll Cardiol, 24, 1468-1474 https://doi.org/10.1016/0735-1097(94)90141-4 (1994).

34. lezzi, R. et al. Foot CT perfusion in patients with peripheral arterial occlusive disease (PAOD): a feasibility study. European journal of radiology 82, e455-464, doi:10.1016/j.ejrad.2012.12.021 (2013).

\section{Figures}


A

Study duration

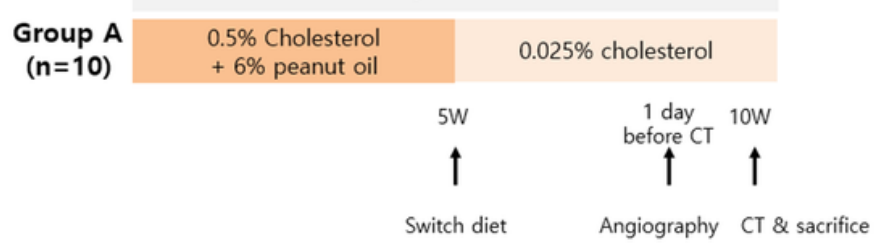

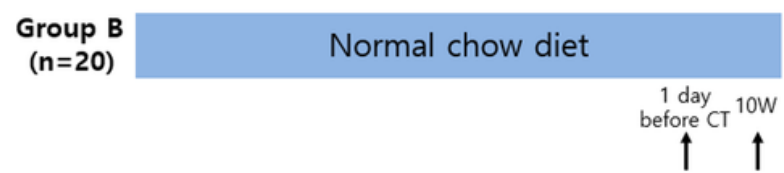

Angiography CT \& sacrifice

B

\begin{tabular}{|c|c|c|c|c|}
\hline $\begin{array}{c}\text { Baseline } \\
\text { perfusion CT }\end{array}$ & $\stackrel{s \min n e t}{\longrightarrow}$ & $\begin{array}{l}\text { Cuff inflation at left thigh } \\
\text { for } 3 \text { minutes followed by } \\
\text { rapid deflation }\end{array}$ & 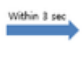 & $\begin{array}{l}\text { Cuff-induced reactive } \\
\text { hyperemia } \\
\text { perfusion CT }\end{array}$ \\
\hline
\end{tabular}

C

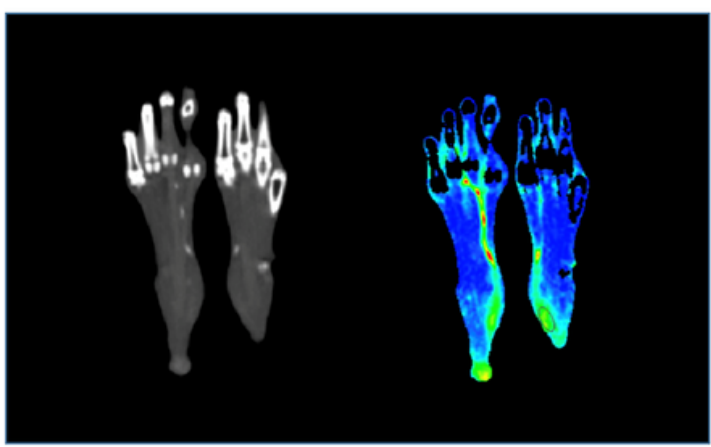

D

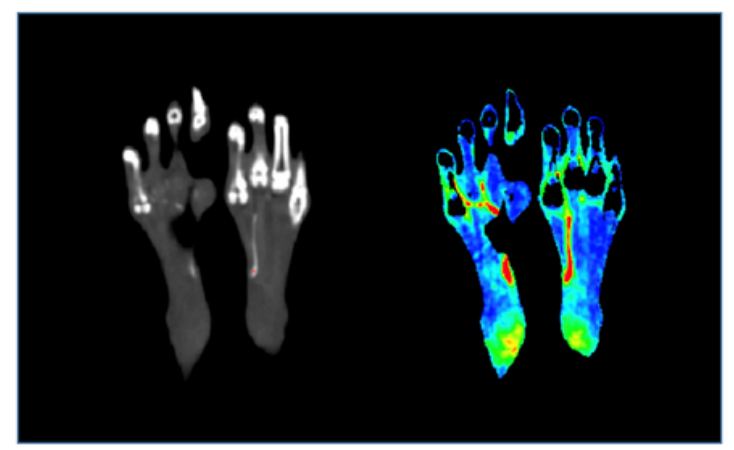

\section{Figure 1}

(a) Study protocol for induction of atherosclerosis. (b) Timeline of CT perfusion protocol. (c) Color-coded perfusion map was reconstructed with perfusion CT. Microperfusion values were measured at the hyperperfusion tissue in the rabbit's heels (center). Region of interest is notated by blue circle on reconstructed image. (d) Tibial artery was measured in the foot, shown in red circle on non-reconstructed CT image. 


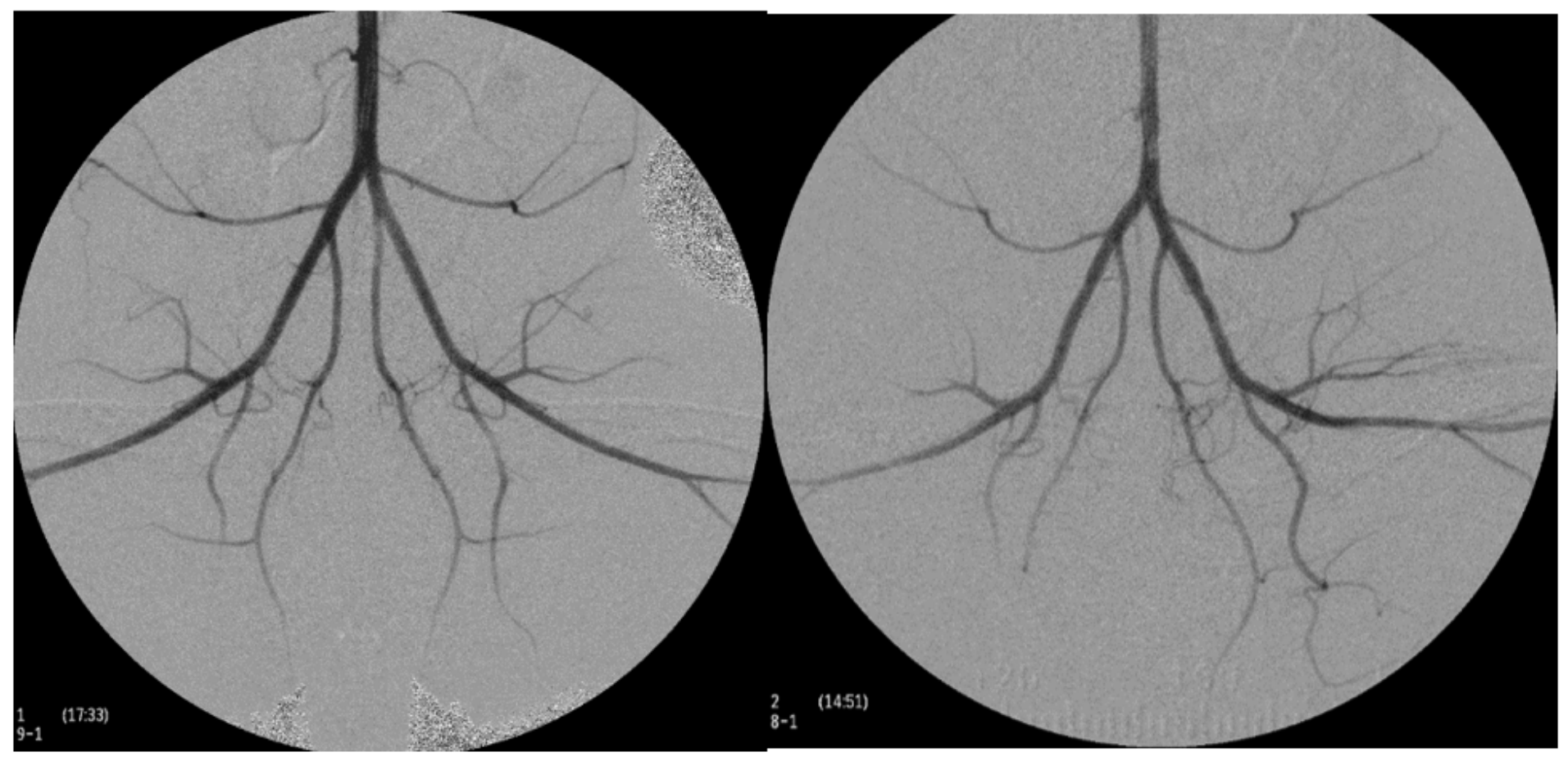

Figure 2

Angiography of healthy rabbits (left) and cholesterol fed rabbits (right) showed no significant stenosis or occlusions in the aortoiliac and femorotibial arteries.

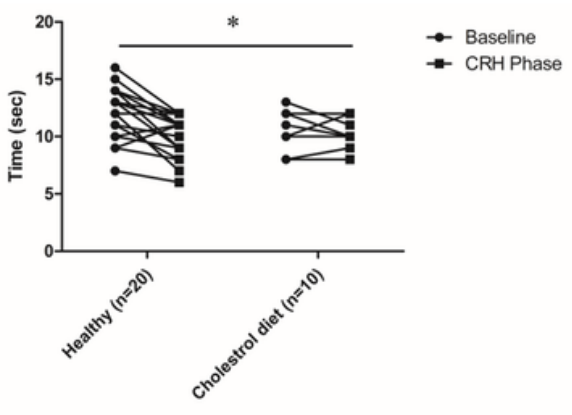

A

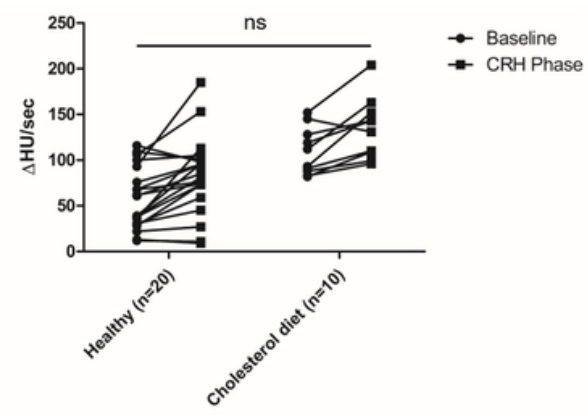

B

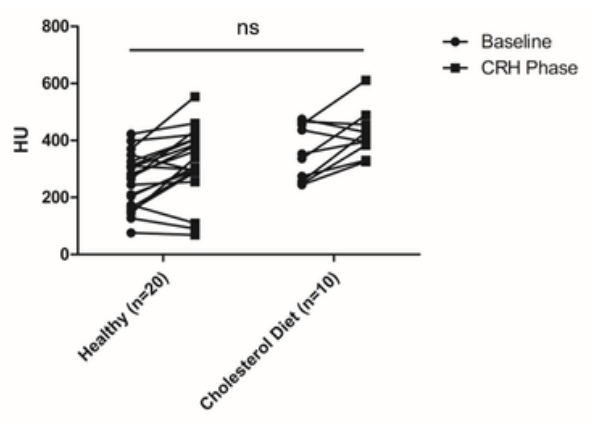

C

Figure 3

(a) Arterial time to peak. At baseline, cholesterol fed rabbits showed increased peak arterial enhancement (PAE) compared to healthy rabbits $(354.6 \pm 96.47 \mathrm{HU}$ vs $255.51 \pm 96.22 \mathrm{HU}, \mathrm{P}<0.001)$. During $\mathrm{CRH}$ phase both groups showed an increased in PAE but intergroup differences were not significant. PAE = Arterial enhancement at $\mathrm{CRH}$ - Arterial enhancement at baseline. Housfield Unit (HU) (b) Arterial slope. Baseline arterial velocity was higher in cholesterol rabbits compared to normal rabbits $(109.30 \pm 25$ vs $57.35 \pm 33.44$ $\mathrm{HU}, \mathrm{P}<0.001$ ). During $\mathrm{CRH}$ phase, there was an increase in slope but no significant intergroup difference $(p=0.977)$. (c) Peak arterial enhancement. Cholesterol-fed rabbits showed no significant arterial time-topeak differences at baseline compared to healthy rabbits $(10.7 \pm 1.82 \mathrm{HU}$ vs $11.65 \pm 2.32 \mathrm{HU}, \mathrm{P}=0.269)$. 
However, arterial TTP showed significant acceleration in healthy rabbits while no such reaction was observed in the cholesterol-fed group $(p=0.025)$.
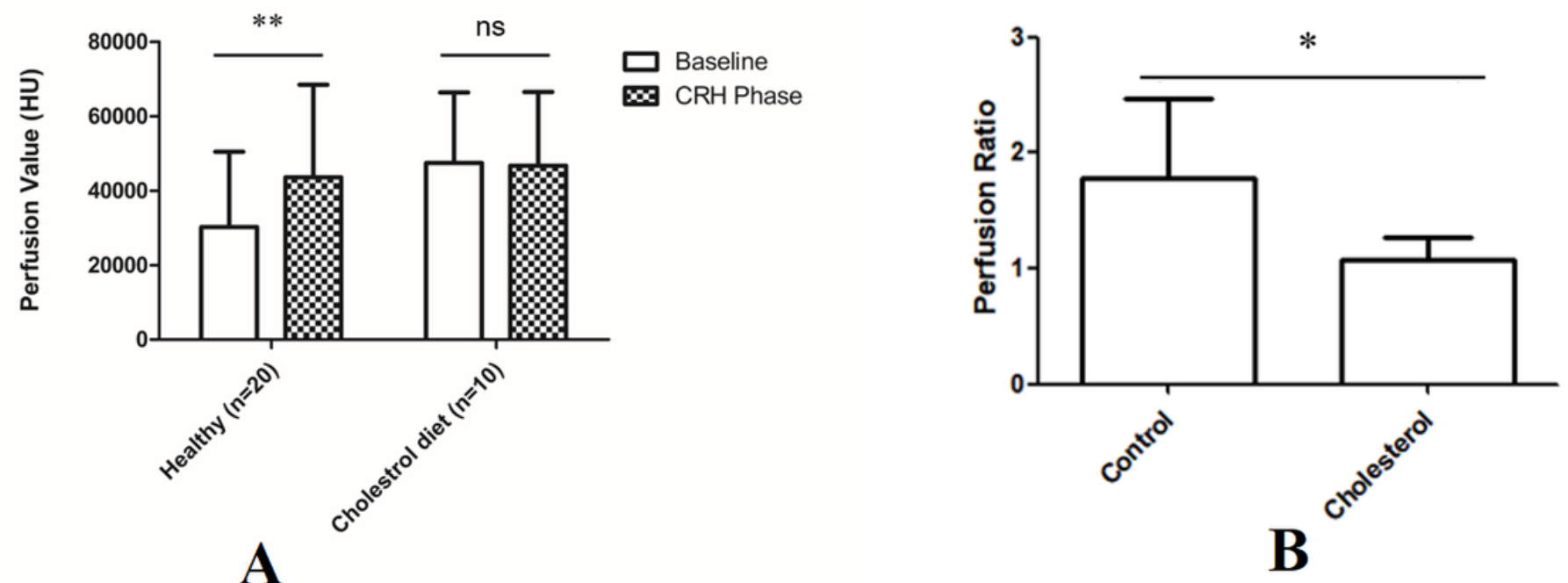

\section{Figure 4}

(a) Perfusion value showed significant increase in the healthy rabbits on $\mathrm{CRH}$ phase $(35040.61 \pm$ 17750.58 to $57582.43 \pm 22585.11 \mathrm{HU} ; \mathrm{P}=0.008$ ) while no such surge was demonstrated in the cholesterol group (47427.59 \pm 18968.08 to $46692.38 \pm 19797.93 \mathrm{HU} ; \mathrm{P}=0.878$ ). (b) Perfusion ratio, the ratio of perfusion at baseline vs. perfusion during $\mathrm{CRH}$ phase, showed significant drop in the cholesterol group compared to control group $(\mathrm{p}=0.014)$.

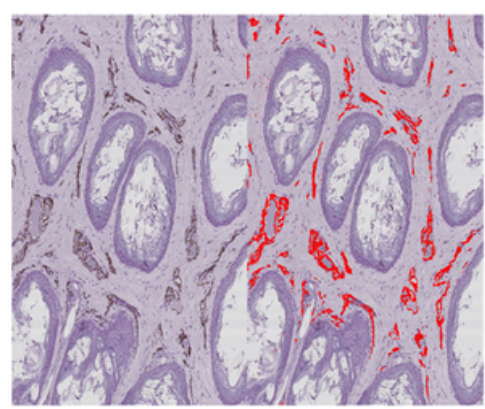

Healthy Rabbit

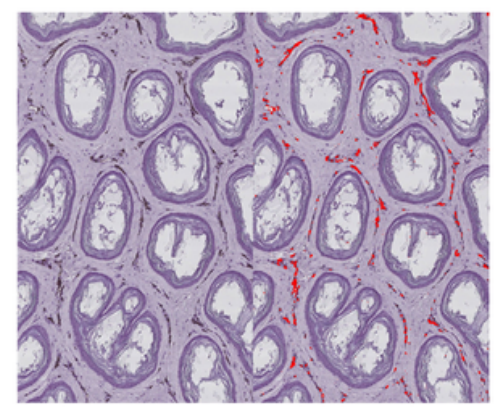

Cholesterol-fed Rabbit

A

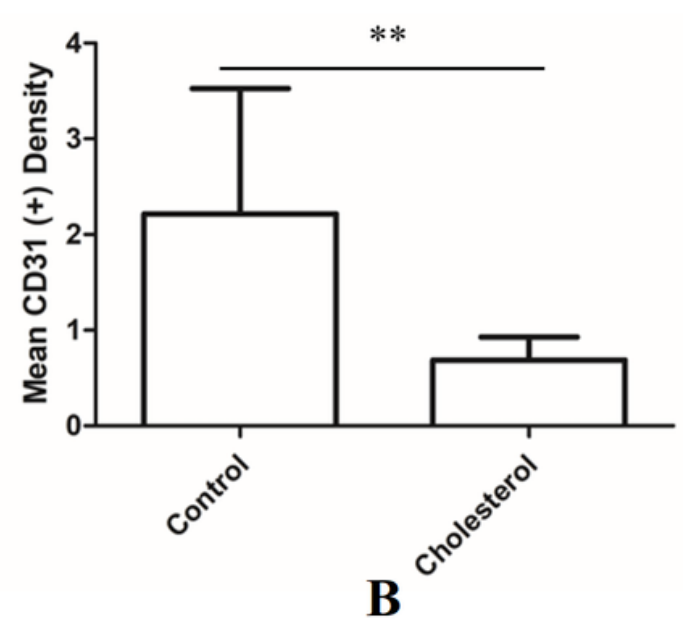

\section{Figure 5}

(a) Cholesterol-fed rabbits showed decreased endothelial cell density as measured by CD 31 expression.

(b) Endothelial cell density as measured by CD 31 showed a lower density in the cholesterol-fed rabbits compared to control $(p<0.001)$. 